УДК 538.958

DOI 10.25205/2541-9447-2019-14-1-63-76

\title{
Оптические плазмонные резонансы в массивах нанокластеров Аu
}

\author{
К. В. Аникин ${ }^{1}$, А. Г. Милёхин ${ }^{1,2}$, Е. Е. Родякина ${ }^{1,2}$, С. Л. Вебер ${ }^{3}$ \\ A. В. Латышев ${ }^{1,2}$, D. R. T. Zahn ${ }^{4}$ \\ ${ }^{1}$ Институт физики полупроводников им. А. В. Ржанова СО РАН \\ Новосибирск, Россия \\ ${ }^{2}$ Новосибирский государственный университет \\ Новосибирск, Россия \\ ${ }^{3}$ Международный томографический иентр СО РАН \\ Новосибирск, Россия \\ ${ }^{4}$ Chemnitz University of Technology, Semiconductor Physics \\ Chemnitz, Germany
}

Аннотация

Работа посвящена исследованию явления локализованного поверхностного плазмонного резонанса (ЛППР) в массивах нанокластеров Аu с помощью оптической спектроскопии отражения. Массивы нанокластеров, имеющих форму цилиндра, диаметр и период которых варьируются в диапазоне 30-150 и 130-200 нм соответственно, были изготовлены на подложках $\mathrm{Si}$ и $\mathrm{Si} / \mathrm{SiO}_{2}$ методом электронно-лучевой литографии. Из сравнения экспериментальных спектров отражения с численно рассчитанными методом конечной разницы во временной области (англ. finite difference time domain, FDTD) были определены структурные параметры модели плазмонных наноструктур, используемые в дальнейшем для расчета спектров поглощения плазмонных структур. Значения частот ЛППР определялись по максимумам спектров поглощения.

Данное исследование выявило сильную зависимость частотного положения ЛППР от размера нанокластеров, расстояния между нанокластерами, а также от толщины слоя $\mathrm{SiO}_{2}$ в нанометровом диапазоне. Особое внимание было уделено наблюдению по спектрам отражения формирования поперечной плазмонной моды, распространяющейся вдоль поверхности подложки и поляризованной перпендикулярно поверхности. Возбуждение данной моды мы связываем с рассеянием электромагнитного поля на соседних нанокластерах.

Предложенный нами метод обеспечивает возможность оперативного определения частотного положения ЛППР по спектрам оптического отражения, что особенно важно в случае непрозрачных подложек (включая подложки $\mathrm{Si}$ и $\mathrm{Si} / \mathrm{SiO}_{2}$ ), для которых измерения оптического поглощения оказывается невозможным. Широкое поле потенциальных применений металлических наноструктур с хорошо контролируемыми плазмонными свойствами включает поверхностно-усиленное инфракрасное поглощение, фотолюминесценцию и комбинационное рассеяние, а также передачу сигнала в кремниевой фотонике.

Ключевые слова

локализованный поверхностный плазмонный резонанс, нанокластеры $\mathrm{Au}$, оптическая спектроскопия отражения, оптическое поглощение

Благодарности

Исследование выполнено при финансовой поддержке РФФИ и Немецкого научно-исследовательского сообщества в рамках проектов РФФИ (проекты № 18-02-00615_а, 19-52-12041 ННИО_а).

Мы благодарим А. Орешонкова и А. Шахраманьяна за помощь в численном моделировании.

С. Л. Вебер благодарит РНФ за поддержку в проведении Фурье-ИК измерений (грант № 17-13-01412)

Для ичтирования

Аникин К. В., Милёхин А. Г., Родякина Е. Е., Вебер С. Л., Латышев А. B., Zahn D. R. T. Оптические плазмонные резонансы в массивах нанокластеров $\mathrm{Au} / /$ Сибирский физический журнал. 2019. Т. 14, № 1. С. 63-76. DOI 10.25205/2541-9447-2019-14-1-63-76

(ㄱ К. В. Аникин, А. Г. Милёхин, Е. Е. Родякина, С. Л. Вебер,

A. В. Латышев, D. R. T. Zahn, 2019 


\title{
Optical Plasmon Resonances in Arrays of Au Nanoclusters
}

\author{
K. V. Anikin ${ }^{1}$, A. G. Milekhin ${ }^{1,2}$, E. E. Rodyakina ${ }^{1,2}$, S. L. Veber ${ }^{3}$ \\ A. V. Latyshev ${ }^{1,2}$, D. R. T. Zahn ${ }^{4}$ \\ ${ }^{1}$ A. V. Rzhanov Institute of Semiconductor Physics SB RAS \\ Novosibirsk, Russian Federation \\ ${ }^{2}$ Novosibirsk State University \\ Novosibirsk, Russian Federation \\ ${ }^{3}$ International Tomographic Centre SB RAS \\ Novosibirsk, Russian Federation \\ ${ }^{4}$ Chemnitz University of Technology, Semiconductor Physics \\ Chemnitz, Germany
}

\section{Abstract}

This work is devoted to the study of the phenomenon of localized surface plasmon resonance (LSPR) in Au nanocluster arrays using optical reflection spectroscopy. Arrays of nanoclusters having the shape of a cylinder, the diameter and period of which vary in the range of 30-150 and 130-200 nm, respectively, were manufactured on Si and $\mathrm{Si} / \mathrm{SiO}_{2}$ substrates by electron beam lithography. From a comparison of experimental reflection spectra with numerically calculated by finite difference time domain method (FDTD), the structural parameters of the model of plasmon nanostructures, used later for the calculation of absorption spectra of plasmon structures, were determined. The LSPR frequencies were determined by the maximum of the absorption spectra.

This study revealed a strong dependence of the LSPR frequency position on the size of nanoclusters, the distance between nanoclusters, and the thickness of the $\mathrm{SiO}_{2}$ layer in the nanometer range. Particular attention was paid to the observation of the reflection spectra of the formation of a transverse plasmon mode propagating along the surface of the substrate and polarized perpendicular to the surface. We associate the excitation of this mode with the scattering of the electromagnetic field on neighboring nanoclusters.

The proposed method provides the possibility of rapid determination of the frequency position of the LSPR in the optical reflection spectra, which is especially important in the case of opaque substrates (including $\mathrm{Si}$ and $\mathrm{Si} / \mathrm{SiO}_{2}$ substrates), for which the measurement of optical absorption is impossible. A wide range of potential applications for metallic nanostructures with well-controlled plasmon properties includes surface-enhanced infrared absorption, photoluminescence, and Raman scattering, as well as signal transmission in silicon Photonics.

Keywords

localized surface plasmon resonance, Au nanoclusters, optical reflection spectroscopy, optical absorption

Acknowledgements

The study was carried out with the financial support of the Russian Foundation for Basic Research and the German Scientific and Research Association within the framework of RFBR projects (projects no. 18-02-00615 a, 19-5212041 NNIO_a).

We thank A. Oreshonkov and A. Shakhramanyan for help in numerical modeling.

S. L. Veber acknowledges RSF (grant no. 17-13-01412) for support of FT-IR meauserements.

For citation

Anikin K. V., Milekhin A. G., Rodyakina E. E., Veber S. L., Latyshev A. V., Zahn D. R. T. Optical Plasmon Resonances in Arrays of Au Nanoclusters. Siberian Journal of Physics, 2019, vol. 14, no. 1, p. 63-76. (in Russ.) DOI 10.25205/2541-9447-2019-14-1-63-76

\section{Введение}

Область плазмоники быстро расширилась за последнее десятилетие благодаря новым разработкам в области нанотехнологии $[1 ; 2]$. Плазмонные структуры на базе металлических нанокластеров ( $\mathrm{Au}, \mathrm{Ag}, \mathrm{Cu}$ и др.), имеющие сфероидальные или более сложные формы, обладают перспективными свойствами для применения в нанозондировании, термоиндуцированном катализе, для визуализации нанообъектов и обнаружения одиночных молекул, однофотонного излучения, нанофотоники, квантовых вычислений и др. [3-8]. Локальное электромагнитное (ЭМ) поле вблизи плазмонных структур отвечает за поверхностное усиленное поглощение, фотолюминесценцию и комбинационное рассеяние (КР) аналитами, расположенными вблизи плазмонных структур. Сильное усиление ЭМ поля в нанометровом масштабе достигается вблизи поверхности плазмонных наноструктур, когда энергия, используемая 
для возбуждения оптического отклика, совпадает с энергией локализованного поверхностного плазмона в плазмонных наноструктурах. При этом энергия последних зависит от структурных параметров плазмонной структуры (размера, формы и расстояния между металлическими наноструктурами), а также от диэлектрических свойств окружающей среды.

Для изготовления ансамблей металлических нанокластеров различных размеров, форм и поверхностной плотности применялись различные методы синтеза [3]. Эти методы условно можно разделить на две группы. Подходы снизу вверх подразумевают самосборку во время процесса формирования, и к ним относятся такие методы, как вакуумное испарение, магнетронное распыление, коллоидная или жидкостная химия. Методы сверху вниз, такие как ионная, электронная, импринт-нанолитография и наносферная литография, требуют частичного удаления материала. Оба подхода имеют свои преимущества и недостатки и применяются в зависимости от конкретной задачи. Методы снизу вверх являются относительно быстрыми и недорогими и позволяют изготавливать нанокластеры, состоящие из различных металлов, на больших площадях различных подложек, но, как правило, эти нанокластеры имеют неоднородные размеры и форму. И наоборот, методы нанолитографии предоставляют уникальную возможность изготовления массивов металлических нанокластеров с точно контролируемыми структурными параметрами (размером, формой и периодом нанокластеров). Однако они требуют много времени, высокой точности позиционирования, сложного и дорогостоящего оборудования. Как следствие, они ограничиваются изготовлением массивов с относительно небольшими площадями.

Металлические наноструктуры, изготовленные на подложках на основе $\mathrm{Si}$, представляют особый интерес в связи с совместимостью процесса их изготовления с кремниевыми технологиями, широко используемыми в микроэлектронике. Существуют хорошо развитые методы осаждения золота на кремниевые подложки, интегрированные в производственную линию микро-/ наноэлектроники. Кроме того, будущее развитие кремниевых технологий включает введение оптических компонентов, изготавливаемых с помощью стандартной технологии кремния. Плазмоника привлекает внимание в качестве возможного инструмента для изготовления нановолноводов и контроля передачи излучения на наноуровне [9; 10]. С этой точки зрения физика плазмонных наноструктур на кремниевых подложках представляет значительный практический интерес.

Обычно положение частоты локализованного поверхностного плазмонного резонанса (ЛППР) можно получить из спектров пропускания / поглощения, экстремумы которых непосредственно соответствуют частотам плазмонного резонанса [11]. Однако такой способ требует прозрачной подложки, например кварца, сапфира или стекла. Действительно, большинство работ, исследующих ЛППР, представляют результаты для металлических нанокластеров, размещенных на прозрачной подложке, например [12; 13], только немногие имеют дело с непрозрачным материалом подложки, таким как кремний [14-18]. Определение частотного положения ЛППР для плазмонных структур на непрозрачных подложках является непростой задачей из-за сложности разделения излучения рассеянного плазмонами и обратного отражения от подложки [19]. Такие методы, как спектроскопия темного поля или спектроскопическая эллипсометрия, могут решить эту проблему, но требуют дорогостоящего оборудования и лабораторных условий. Поэтому продолжаются поиски надежного, недорогого и простого в применении метода, потенциально применимого в процессе перспективного производства.

В данной работе мы используем оптическую спектроскопию отражения для исследования плазмонных свойств массивов нанокластеров $\mathrm{Au}$, изготовленных на подложках $\mathrm{Si} / \mathrm{SiO}_{2}$, с различными размерами и расстояниями между нанокластерами. Показана сильная зависимость энергии ЛППР от изменения толщины слоя $\mathrm{SiO}_{2}$ в нанометровом диапазоне за счет взаимодействия с подложкой $\mathrm{Si}$. Кроме того, рассеяние под прямым углом от соседних нанокластеров возбуждает поперечную моду ЛПП в обсуждаемых структурах. 
На основе сравнения экспериментальных спектров отражения с численно моделируемыми определены модельные структурные параметры плазмонных наноструктур. С помощью этих параметров модели рассчитаны спектры поглощения плазмонных структур после вычитания поглощения подложки. Максимумы в расчетных спектрах поглощения плазмонных образцов использованы для определения энергий ЛППР в массивах нанокластеров $\mathrm{Au}$ на непрозрачных подложках. Наши результаты согласуются с литературными данными, полученными ранее альтернативными методами для нанокластеров Аu идентичной формы на различных подложках [11-17].

\section{Экспериментальная часть}

Периодические массивы нанокластеров Аu на подложках (001)-Si с естественным оксидом кремния или специально нанесенным слоем $\mathrm{SiO}_{2}$ были изготовлены методом прямой электронно-лучевой литографии (Raith-150, Германия) на 130-нм пленке фоторезиста (полиметилметакрилат 950 к), нанесенной методом центрифугирования. Термический оксид $\left(1000{ }^{\circ} \mathrm{C}\right) \mathrm{SiO}_{2}$ толщиной 77 нм на подложке $\mathrm{Si}\left(\mathrm{Si} / \mathrm{SiO}_{2}\right.$ (77нм) подложка), применяемый в настоящем исследовании, был получен в результате травления подложки $\mathrm{Si}$, покрытой 600 нм слоем $\mathrm{SiO}_{2}$ в растворе $\mathrm{HF}$ в $\mathrm{H}_{2} \mathrm{O}$ (в объемном соотношении $2: 5$ ). Толщина слоя $\mathrm{SiO}_{2}$ определялась с помощью спектроскопической эллипсометрии.

Для изготовления плазмонных структур методом вакуумного испарения на нанопрофилированный положительный фоторезист наносилась пленка Аu 40 нм с подслоем Тi 5 нм для лучшей адгезии. Окончательно фоторезист снимался в dimethylformamide по технологии «взрыва», приводя к формированию массивов нанокластеров Аu. В результате были изготовлены плазмонные подложки из 4 различных областей с массивами нанокластеров $\mathrm{Au} \mathrm{c}$ периодами 130, 150, 200 и 250 нм. Каждая область представляет собой 28 квадратных решеток размером $30 \times 30$ мкм $^{2}$ с различными диаметрами нанокластеров $\mathrm{Au}(30-150$ нм) для каждой решетки. СЭМ-изображения массивов нанокластеров $\mathrm{Au}$ были получены с использованием системы Raith-150 при ускоряющем напряжении 10 кВ, диафрагме 30 мм и рабочем расстоянии 6 мм.

Разброс размеров нанокластеров оценивался с помощью программного обеспечения для обработки изображений Gwyddion. Из-за флуктуации размеров нанокластеров золота, образующихся в процессе испарения, точность определения размеров нанокластеров и зазоров между соседними нанокластерами составляла около \pm 5 нм.

Спектры отражения массивов Au измерялись в конфигурации обратного отражения с использованием спектрометра Bruker Vertex 80v FT-IR, поставляемого с микроскопом Hyperion 2000 в диапазоне от 400-900 нм и оснащенного 40-кратным объективом (WD = $=0,65 \mathrm{MM}, \mathrm{NA}=0,65)$. Для полного покрытия области образца диаметр пучка на образце был выбран равным 30 мкм. В качестве эталона использовалось отражение от той же подложки $\mathrm{Si}$ без массивов нанокластеров Аu. Для возбуждения использовалось неполяризованное излучение.

\section{Результат и обсуждение}

Рисунок 1 представляет типичные СЭМ-изображения массивов нанокластеров Аu различного диаметра, изготовленных на подложках $\mathrm{Si} / \mathrm{SiO}_{2}$. С помощью этих изображений были определены структурные параметры массивов нанокластеров, такие как диаметр, форма кластера и период. Кластеры Аu имеют хорошо определенную округлую форму с точностью \pm 5 нм. Высокая точность изготовления образцов обеспечивается характеристиками литографического процесса. Эффект кластеризации Аи и характер процесса изготовления структур приводит к тому, что минимальный размер кластера не может быть менее 10 нм. Эта же причина отвечает за отличия формы нанокластеров Аu от цилиндрической. Тень круглой формы 
вокруг нанокластеров $\mathrm{Au}$, смещенная от центров нанокластеров $\mathrm{Au}$ примерно на 5 нм, является остатком слоя Ti, связанного с различной геометрией источников распыления Ti и $\mathrm{Au}$.

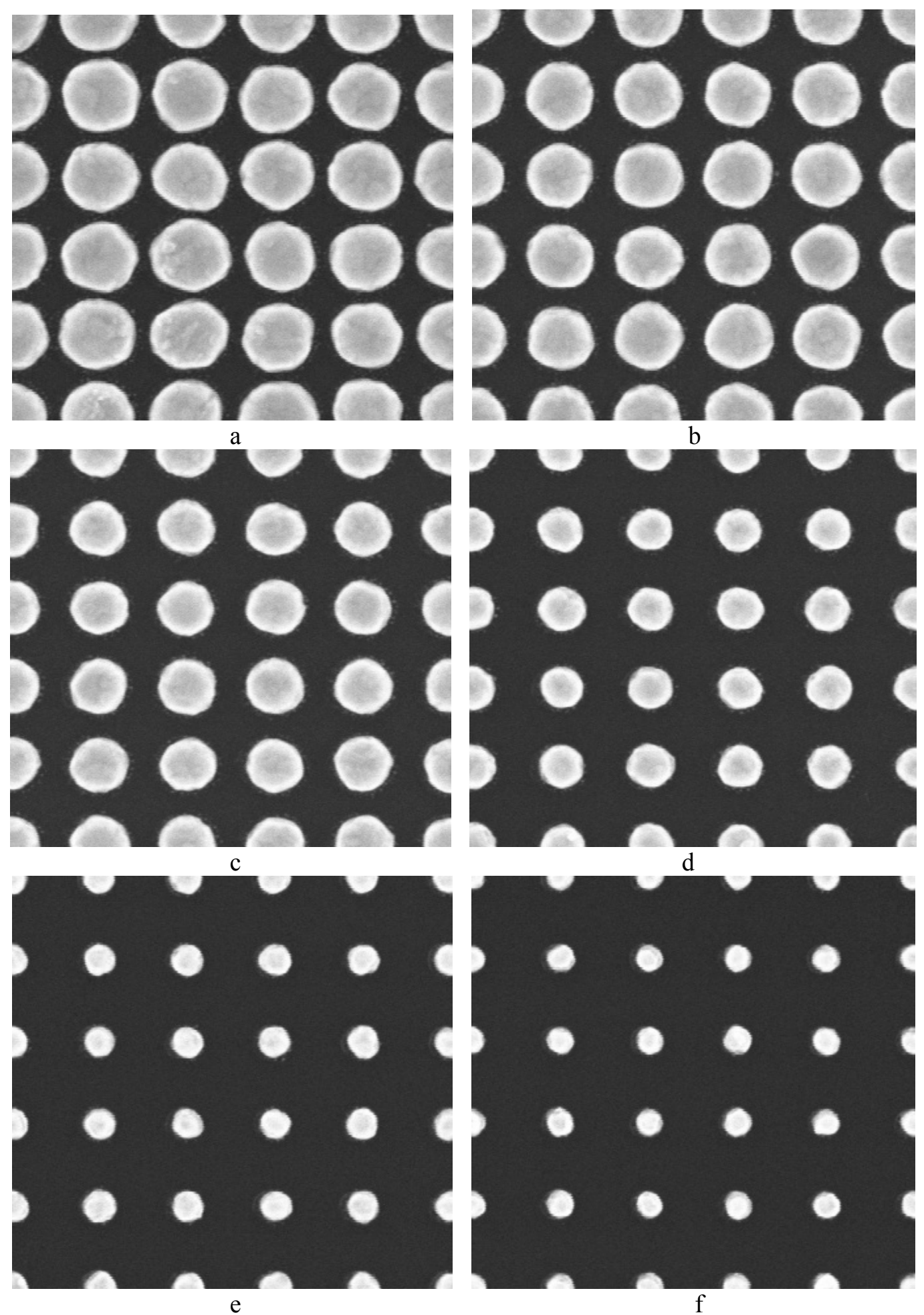

Рис. 1. Типичные СЭМ-изображения нанокластеров Аu разного размера на подложке $\mathrm{Si} / \mathrm{SiO}_{2}$ с периодом 150 нм

Fig. 1. Typical SEM images of Au nanoclusters of different sizes on a $\mathrm{Si} / \mathrm{SiO}_{2}$ substrate with a period of $150 \mathrm{~nm}$ 


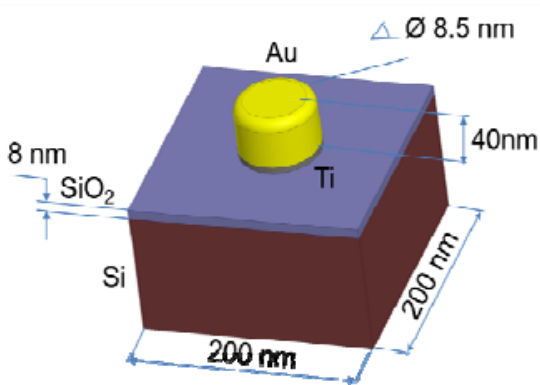

a

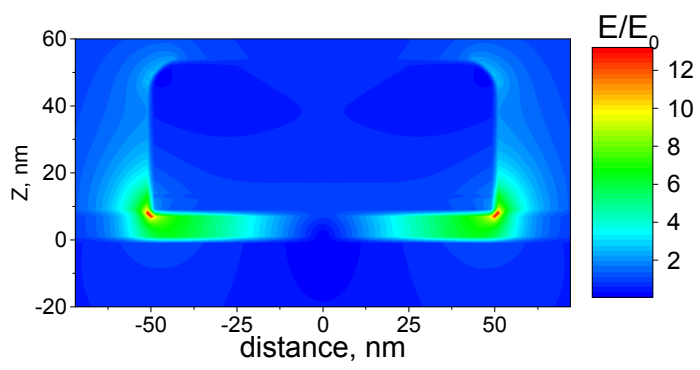

$\mathrm{c}$

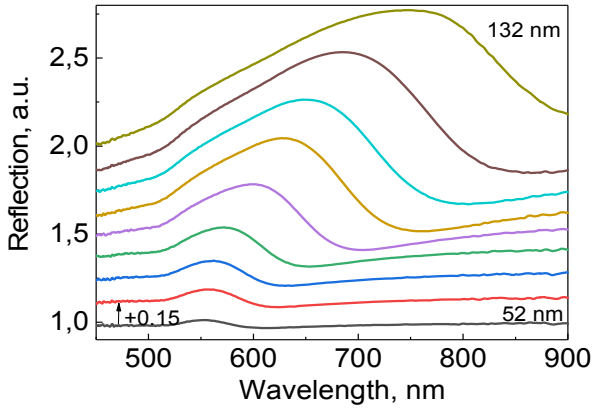

e

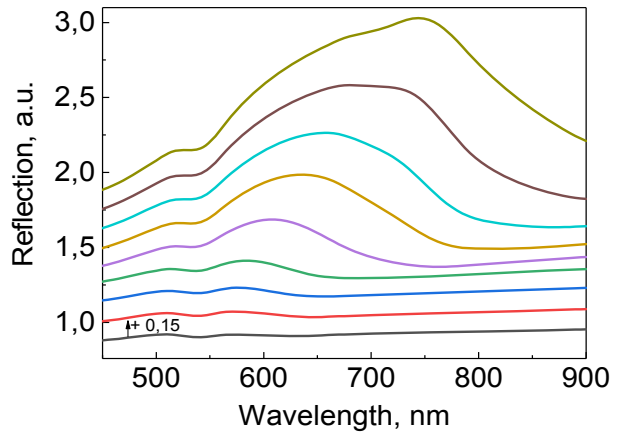

$\mathrm{h}$

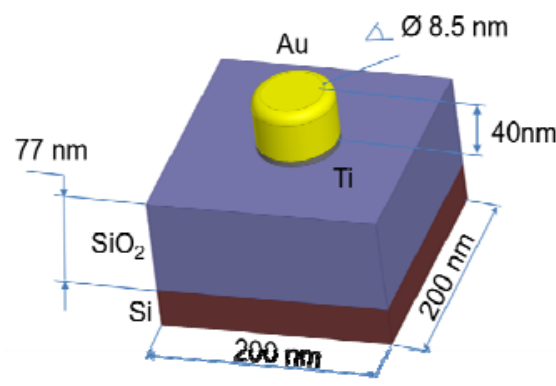

b

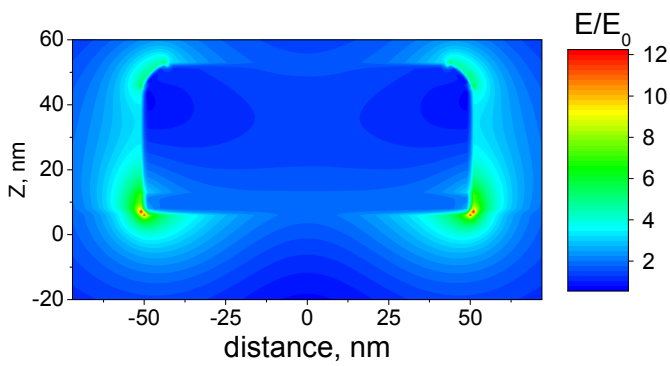

d

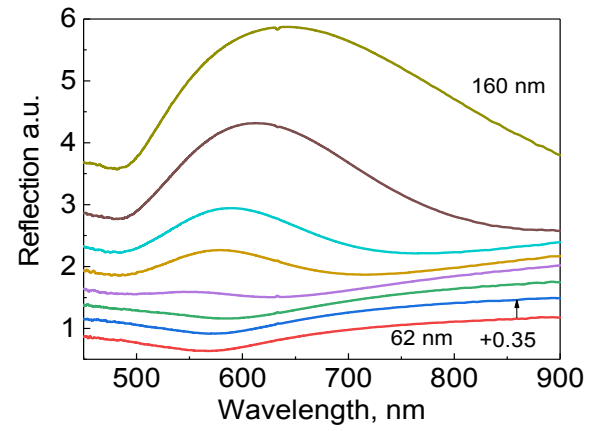

$\mathrm{f}$

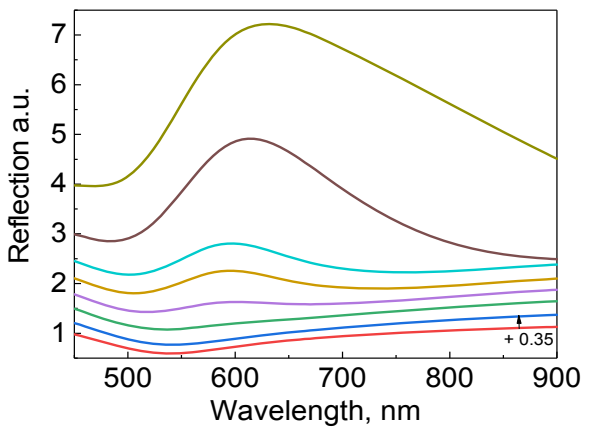

g

Puc. 2. Элементарные ячейки $(a, b)$ для структур с массивами нанокластеров $\mathrm{Au}$ на подложках $\mathrm{Si} / \mathrm{SiO}_{2}(8 \mathrm{Hм})$ и $\mathrm{Si} / \mathrm{SiO}_{2}$ (77 нм) соответственно. Расчетное электрическое поле вблизи нанокластеров Аи диаметром 100 нм на подложках (c) Si / $\mathrm{SiO}_{2}$ (8 нм) и (d) $\mathrm{Si} / \mathrm{SiO}_{2}$ (77 нм) (при 720 и 571 нм соответственно). Экспериментальные (e, $f)$ и рассчитанные $(h, g)$ спектры отражения для нанокластеров (диаметры 52, 58, 61, 68, 83, 96, 105, 117 и 132 нм на $\mathrm{Si} / \mathrm{SiO}_{2}$ (8 нм) и 62, 70, 78, 86, 94, 98, 124 и 160 нм на $\mathrm{Si} / \mathrm{SiO}_{2}$ (77 нм) соответственно

Fig. 2. Unit cells $(a, b)$ for structures with arrays of Au nanoclusters on $\mathrm{Si} / \mathrm{SiO} 2(8 \mathrm{~nm})$ and $\mathrm{Si} / \mathrm{SiO} 2(77 \mathrm{~nm})$ substrates, respectively. The calculated electric field near $\mathrm{Au}$ nanoclusters $100 \mathrm{~nm}$ in diameter on $(c) \mathrm{Si} / \mathrm{SiO} 2(8 \mathrm{~nm})$ and (d) $\mathrm{Si} / \mathrm{SiO} 2(77 \mathrm{~nm})$ substrates (at 720 and $571 \mathrm{~nm}$, respectively). The experimental $(e, f)$ and calculated $(h, g)$ reflection spectra for nanoclusters (diameters 52, 58, 61, 68, 83, 96, 105, 117 and $132 \mathrm{~nm}$ on Si / SiO2 (8 nm) and 62, 70, 78, 86, 94, 98, 124 and $160 \mathrm{~nm}$ on $\mathrm{Si} / \mathrm{SiO} 2(77 \mathrm{~nm})$, respectively 
Толщина слоя $\mathrm{SiO}_{2}$ на подложках $\mathrm{Si} / \mathrm{SiO}_{2}$ определялась с помощью спектральной эллипсометрии. В случае подложек $\mathrm{Si}$ измерения дают толщину слоя оксида кремния $(7,5 \pm 0,5) \mathrm{Hм}$ (далее обозначается подложкой $\mathrm{Si} / \mathrm{SiO}_{2}(8 \mathrm{Hм})$ ), которая превышает ожидаемое значение для естественных оксидов (около 3-4 нм) за счет дальнейшего окисления в процессе изготовления нанокластеров $\mathrm{Au}$. Для подложек, обозначенных как $\mathrm{Si} / \mathrm{SiO}_{2}$ (77 нм), толщина слоя $\mathrm{SiO}_{2}$ составляет около $(77 \pm 1)$ нм.

На рис. 2 приведены схематические изображения структур с нанокластерами Аu на подложках $\mathrm{Si} / \mathrm{SiO}_{2}(8 \mathrm{нм})$ и $\mathrm{Si} / \mathrm{SiO}_{2}(77 \mathrm{Hм})$, а также соответствующие экспериментальные спектры отражения, рассчитанные с использованием модели, рассмотренной далее.

Для определения положения ЛППР распределение ЭМ поля в образцах численно моделировалось методом конечной разности во временной области (англ. finite difference time domain, FDTD) (рис. 2, $c, d$ ) с использованием коммерческого пакета Lumerical (Ванкувер, Канада). Для моделирования исходные структурные параметры массивов нанокластеров были взяты из измерений СЭМ и эллипсометрии, а также из данных технологического процесса. Эти параметры были дополнительно подогнаны для лучшего совпадения экспериментальных и расчетных спектров. Диэлектрические функции $\mathrm{Au}, \mathrm{Ti}, \mathrm{Si}$ и $\mathrm{SiO}_{2}$ были взяты из литературы [20-23]. При моделировании обе подложки $\mathrm{Si} / \mathrm{SiO}_{2}$ моделировались как подложки $\mathrm{Si} \mathrm{c} \mathrm{различной} \mathrm{толщиной} \mathrm{слоя} \mathrm{SiO}_{2}$. Радиус кривизны диска Аu был принят во внимание по результатам, представленным в [19].

Как видно из рис. $2, c, d$, электрическое поле на длине волны ЛППР 720 и 571 нм соответственно для нанокластеров Аu с диаметром 100 нм на $\mathrm{Si} / \mathrm{SiO}_{2}$ (8 нм) и $\mathrm{Si} / \mathrm{SiO}_{2}$ (77 нм) подложках преимущественно локализуются на краях нанокластера. Распределение и максимальная амплитуда электрического поля $\left(E / E_{0} \sim 12\right)$ практически одинаковы для обоих случаев.

Для обоих типов подложек рис. $2, e, f$ демонстрируют выраженные изменения в спектрах отражения при изменении размера кластера. В случае подложки $\mathrm{Si} / \mathrm{SiO}_{2}$ (8 нм) (см. рис. 2, e) интенсивность отражения увеличивается, в то время как максимум отражения монотонно смещается в красную спектральную область с увеличением размера кластера. Отметим, что общая форма спектров сохраняется. В случае $\mathrm{Si} / \mathrm{SiO}_{2}$ (77 нм) подложки спектры отражения (см. рис. $2, f$ ) для малых размеров кластеров демонстрируют выраженные минимумы, которые превращаются в максимумы при диаметре около 90 нм. Сложное поведение спектров отражения, которое определяется суперпозицией обратного рассеяния от наноструктур $\mathrm{Au}$ и отражением от подложки $\mathrm{Si}$, препятствует прямому определению положения ЛППР из спектров отражения. Вычитание отражения подложки из измеренных спектров затруднено, так как отражение от подложки модифицируется наличием нанокластеров $\mathrm{Au}$.

Расчетные спектры нанокластеров Аи диаметром от 52 до 132 нм (62 и 160 нм), изготовленных на подложках $\mathrm{Si} / \mathrm{SiO}_{2}$, представлены на рис. $2, h, g$, где высота кластеров $\mathrm{Au}$, подслоя Ті и радиус кривизны составляют 40,5 и 4,25 нм соответственно. Размер нанокластеров $\mathrm{Au}$, использованных при расчете, соответствует данным СЭМ-микроскопии соответствующих структур. Как видно из рис. 2 , общая форма и спектральное положение основных линий расчетного и экспериментального спектров совпадают.

Для получения наилучшего соответствия с экспериментальными данными использовалась модель, изображенная на рис. 2, $a, b$. Использовались три параметра оптимизации, которые наиболее сильно влияют на спектры отражения, а именно: толщина слоя $\mathrm{SiO}_{2}$, кривизна края диска и толщина слоя Тi. Такие параметры, как шероховатость поверхностей $\mathrm{Au}, \mathrm{Si}, \mathrm{SiO}_{2}$, изменение высоты диска и изменение диэлектрической проницаемости $\mathrm{SiO}_{2}$, слабо влияют на расчетные спектры по сравнению с параметрами подгонки, упомянутыми выше, и были исключены из рассмотрения. Небольшое несоответствие экспериментального и расчетного спектров может быть объяснено следующими факторами: (I) частичная диффузия подслоя Тi в слой $\mathrm{SiO}_{2}$, модифицирующая диэлектрическую проницаемость $\mathrm{SiO}_{2}$ (этот эффект исследован в [24]); (II) смещение между пространственным расположением центров оставшегося подслоя Ті и кластера $\mathrm{Au}$; (III) общая неоднородность на нанометровом масштабе структур- 
ных параметров образца; (IV) ограниченная апертура объектива микроскопа, используемого для регистрации спектров отражения. Эти четыре пункта в модели не учитывались.

Следующим шагом было использование этой модели для расчета спектров поглощения структур и получения частотного положения ЛППР. Поскольку спектры поглощения определяются безызлучательной релаксацией поверхностных плазмонов, причем максимумы поглощения ЛППР совпадают с максимумами спектров рассеяния (излучательная релаксация ЛПП) $[11 ; 12]$, то для определения положения длины волны ЛППР можно использовать максимумы поглощения. Для расчета спектров поглощения использовался следующий подход.

Принимая во внимание закон сохранения энергии $1=R+T+(A+S)$, где $R-$ коэффициент отражения, $T$ - коэффициент пропускания, $A$ - коэффициент поглощения, $S$ - доля рассеянного излучения, $(A+S)$ - коэффициент экстинкции, и периодические граничные условия для используемой в расчетах элементарной ячейки (см. рис. $2, a, b)$, можно написать выражение для коэффициента поглощения:

$$
A=1-\left(R+S_{\text {back }}\right)-\left(T+S_{\text {front }}\right),
$$

где $S_{\text {back }}$ и $S_{\text {front }}$ - доля ЭМ излучения, рассеиваемая в верхнее и нижнее полупространство соответственно. Из расчета мы получаем непосредственно $R+S_{\text {back }}$ и $T+S_{\text {front }}$ как общее ЭМ поле, распространяющееся в верхнее и нижнее полупространство соответственно. Пропускание и рассеяние в нижнее полупространство $\left(T+S_{\text {front }}\right)$ определялось из расчета потока мощности ЭМ поля через плоскость, помещенную в объеме Si на 100 нм ниже границы раздела $\mathrm{Si} / \mathrm{SiO}_{2}$. Расстояние 100 нм было выбрано для устранения влияния эффектов ближнего поля нанокластеров Au.

На рис. $3, a, b$ демонстрируются спектры поглощения для структур, схематически показанных на рис. $2, a, b$, рассчитанных с использованием описанного выше подхода. Доминирующие максимумы поглощения, наблюдаемые в спектрах нанокластеров Аu на подложках $\mathrm{Si} / \mathrm{SiO}_{2}(8$ нм) (см. рис. 3, $a$ ), смещаются от 580 до 780 нм с увеличением размера нанокластера Au с 52 до 132 нм и соответствует максимумам отражения, представленным на рис. $2, h$. Увеличение поглощения ниже 500 нм (см. рис. 3, a) обусловлено межзонными d-s-переходами в золоте [25].

Следует отметить относительно слабый максимум при 530 нм с почти одинаковой интенсивностью для структур с различным размером нанокластеров (см. рис. $3, a$ ). По нашим расчетам, представленным на рис. 4 , эта особенность становится более выраженной с увеличением угла падения света, поэтому мы интерпретируем ее как поперечную ЛППР-моду, индуцированную рассеянием от соседних нанокластеров и поляризованную перпендикулярно поверхности образца. В работах $[4 ; 26 ; 27]$ уже наблюдалась активация поперечной моды за счет взаимодействия соседних кластеров. Для более детального изучения данного эффекта мы рассчитали распространение ЭМ возмущения вдоль плоскости образца, представляющего собой золотые диски, выстроенные в линию, аналогичные тем, что мы изучаем в данной работе. Для исключения эффекта подложки мы моделировали нанодиски в вакууме. Результаты расчетов представлены на рис. 5. Как видно из рис. 5, при возбуждении только одного диска (выделен серым прямоугольником) возбуждение распространяется вдоль поверхности подложки на расстояния, значительно превышающие диаметр диска. Это означает, что если расстояние между нанокластерами сравнимо с размером нанокластера, то необходимо учитывать взаимодействие между ними. Спектры поглощения нанокластеров $\mathrm{Au}$ на подложке $\mathrm{Si} / \mathrm{SiO}_{2}$ (77 нм) показывают более слабое красное смещение максимума ЛППР с увеличением размера нанокластера $\mathrm{Au}$ (см. рис. $3, b$ ). При размерах нанокластера более 90 нм в спектрах поглощения нанокластеров $\mathrm{Au}$ на подложке $\mathrm{Si} / \mathrm{SiO}_{2}$ (77 нм) начинает доминировать поперечная мода, обусловленная спектральным перекрытием продольной и поперечной мод ЛППР. Этот эффект вызывает синий сдвиг максимума поглощения для нанокластеров $\mathrm{Au}$ размером выше 90 нм (см. рис. $3, b$ ). 


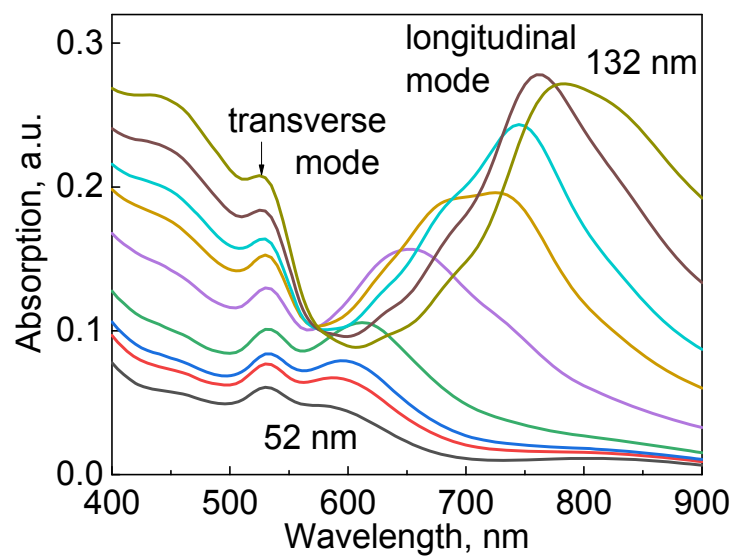

a

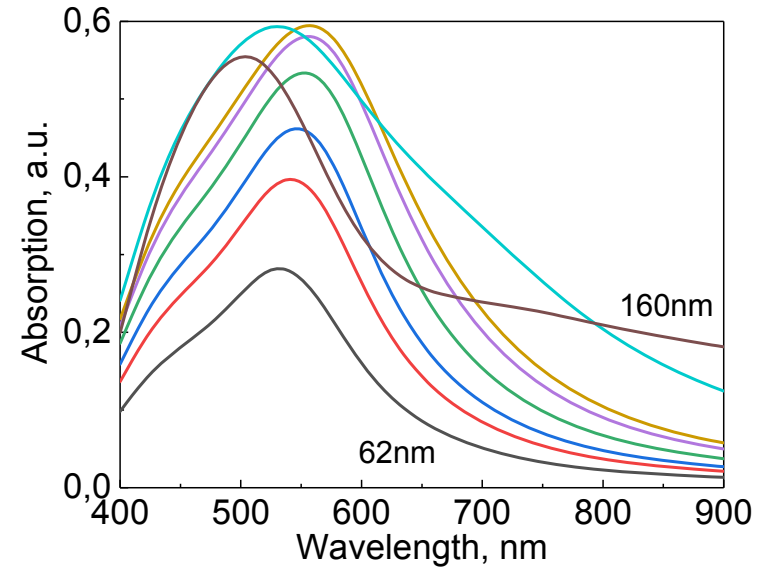

b

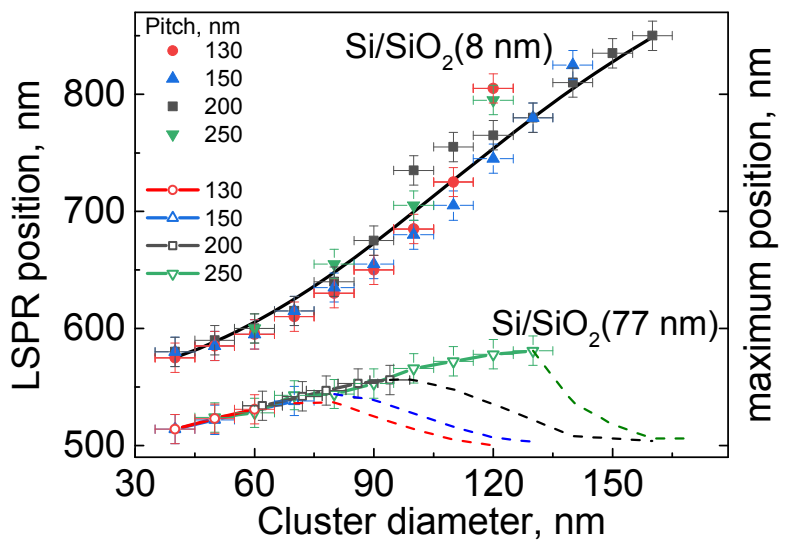

c

Puc. 3. Рассчитанные спектры поглощения массивов нанокластеров $\mathrm{Au}$ на подложках (a) $\mathrm{Si}_{1} \mathrm{SiO}_{2}(8 \mathrm{нм})$ и $(b) \mathrm{Si} / \mathrm{SiO}_{2}$ (77 нм) с такими же структурными параметрами, как показано на рис. 2; (c) спектральное положений максимумов, полученных из спектров поглощения, показанных на рис. $3, a, b$ (сплошные и пунктирные линии) и положение ЛППР (сплошные линии) для кластеров Аu с различными периодами на разных подложках. Сплошные линии изображены для удобства восприятия. Пунктирные линии показывают положение максимума поглощения для случаев, где определение энергий ЛППР затруднительно

Fig. 3. The calculated absorption spectra of arrays of Au nanoclusters on substrates $(a) \mathrm{Si} / \mathrm{SiO}_{2}(8 \mathrm{~nm})$ and $(b) \mathrm{Si} / \mathrm{SiO}_{2}$ $(77 \mathrm{~nm})$ with the same structural parameters as shown in Fig. 2; and (c) The spectral positions of the maxima obtained from the absorption spectra shown in Fig. 3, $a, b$ (solid and dashed lines) and the position of the LPR (solid lines) for Au clusters with different periods on different substrates. Solid lines are shown for convenience. Dotted lines indicate the position of the absorption maximum for cases where the determination of the LPRR energies is difficult

Puc. 4. Спектры поглощения массива нанокластеров $\mathrm{Au}$ диаметром 70 нм и периодом 200 нм на $\mathrm{Si} / \mathrm{SiO}_{2}$ (8 нм) подложке в зависимости от угла падения света $\left(10,30,40,50\right.$ и $\left.60^{\circ}\right)$

Fig. 4. The absorption spectra of an array of Au nanoclusters with a diameter of $70 \mathrm{~nm}$ and a period of $200 \mathrm{~nm}$ on a $\mathrm{Si} / \mathrm{SiO}_{2}(8 \mathrm{~nm})$ substrate, depending on the angle of incidence of light $(10,30,40$, 50 and 60 degrees)

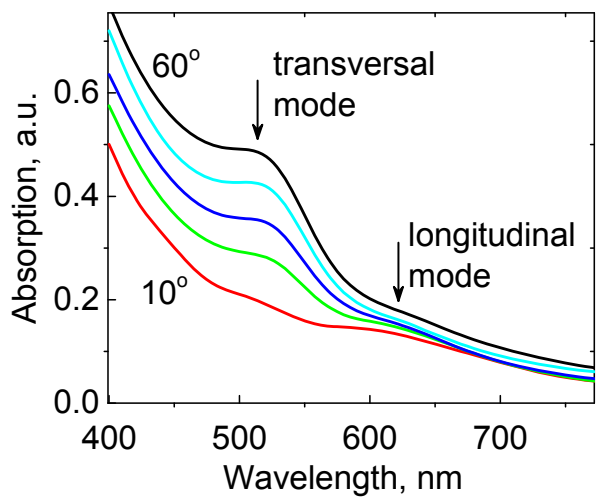

ISSN 2541-9447 


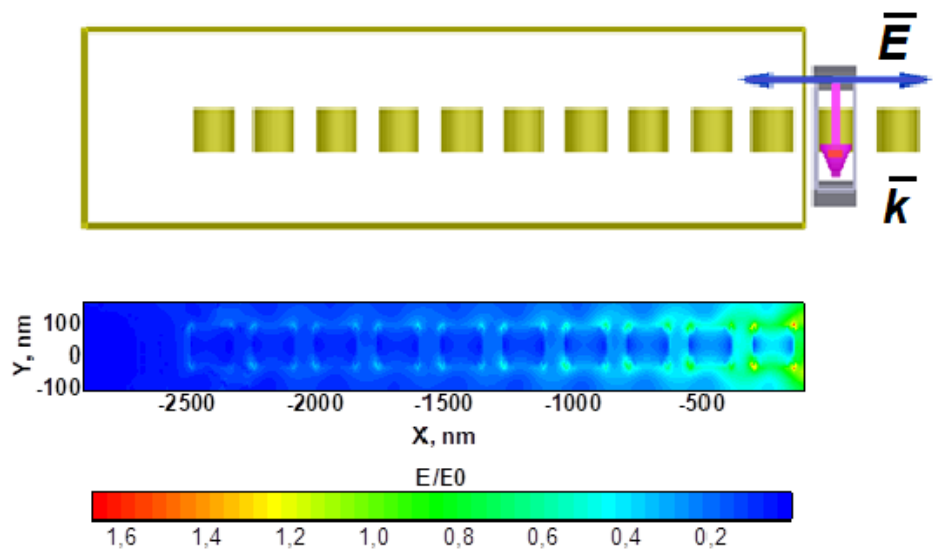

Puc. 5. Модель линейной цепочки нанокластеров $\mathrm{Au}$ диаметром 130 нм и периодом 200 нм (вверху, вид сбоку) и соответствующее распределение электрического поля (внизу)

Fig. 5. Model of a linear chain of Au nanoclusters with a diameter of $130 \mathrm{~nm}$ and a period of $200 \mathrm{~nm}$ (above, side view) and the corresponding distribution of the electric field (below)

Следует отметить, что мы выбрали толщину слоя $\mathrm{SiO}_{2} 77$ нм, чтобы получить дополнительное усиление поля за счет интерференции в тонкой пленке оксида, следуя результатам, представленным в [28]. Условия деструктивной интерференции для слоя $\mathrm{SiO}_{2}$ толщиной 77 нм удовлетворяются при 507 нм и приводят к увеличению поглощения в этой спектральной области. В результате форма спектров поглощения нанокластеров $\mathrm{Au}$ на подложках $\mathrm{Si} / \mathrm{SiO}_{2}$ (77 нм) определяется вкладами четырех эффектов: продольного и поперечного режимов ЛППР, межзонного поглощения Аи и интерференции в оксидном слое.

Спектральное положение ЛППР, полученные из спектров поглощения структур, сформированных на подложках $\mathrm{Si} / \mathrm{SiO}_{2}$, в зависимости от размеров нанокластеров $\mathrm{Au}$ для различных периодов (130-250 нм), показаны на рис. 3, c. Как видно, красное смещение положения ЛППР с увеличением размера нанокластера наиболее выражено для подложки $\mathrm{Si} / \mathrm{SiO}_{2}$ (8 нм). Это можно объяснить более высокой усредненной диэлектрической проницаемостью локальной среды для нанокластеров $\mathrm{Au}$ на подложке. Полученная зависимость положения ЛППР от размера кластера для структур, сформированных на подложке $\mathrm{Si} / \mathrm{SiO}_{2}$ (8 нм), хорошо соответствует нашим предыдущим результатам, полученным по данным спектральной эллипсометрии и моделирования в [16] и измерений в режиме темного поля в [15]. Что касается кластеров $\mathrm{Au}$ на подложке $\mathrm{Si} / \mathrm{SiO}_{2}(77$ нм), то мы сравнивали наши результаты с литературными данными для кластеров Аu на прозрачных подложках $\mathrm{SiO}_{2}$. Положение ЛППР как функция размера нанокластера Аu ведет себя аналогично описанному, например, в [29; 30] для кластеров идентичной высоты. Это подтверждает правильность подхода, использованного нами для расчета.

Отметим, что рассчитанные спектры поглощения выявляют максимумы, приписываемые модам ЛППР, возникающим на более высоких длинах волн (см. рис. $3, a, b$ ), чем в спектрах отражения (см. рис. $2, e, f)$. Этот факт подтверждает важность предлагаемого подхода для определения положения ЛППР, в то время как прямое извлечение энергии ЛППР из экспериментальных спектров отражения приведет к переоценке энергии ЛППР.

При сравнении положения ЛППР от размера нанокластеров $\mathrm{Au}$, сформированных на подложках $\mathrm{Si} / \mathrm{SiO}_{2}(8 \mathrm{Hм})$ и $\mathrm{Si} / \mathrm{SiO}_{2}(77 \mathrm{Hм})$ (см. рис. $\left.3, c\right)$, можно увидеть возможность на- 
стройки положения ЛППР в диапазоне 530 до 800 нм при изменении толщины слоя $\mathrm{SiO}_{2}$. Отметим, что положение ЛППР в большинстве случаев совпадает с максимумами поглощения, представленными на рис. 2. Однако для относительно больших нанокластеров $\mathrm{Au}$ на подложке $\mathrm{Si} / \mathrm{SiO}_{2}$ (77 нм) (при спектральном перекрытии продольной и поперечной мод ЛППР) определение положения ЛППР по спектрам поглощения практически невозможно. В связи с этим, на рис. 3, $c$ показан синий сдвиг максимального положения поглощения с увеличением размера кластера, обозначенного пунктирными линиями.

Разработанный в настоящем исследовании подход открывает путь к созданию нанокластеров $\mathrm{Au}$ со структурными параметрами, обеспечивающими требуемую энергию ЛППР для дальнейшего применения.

\section{Вывод}

Представлен подход, позволяющий на основе анализа спектров отражения определять энергию ЛППР для нанокластеров различного размера, формируемых на непрозрачных подложках $\mathrm{Si} / \mathrm{SiO}_{2}$. Рассчитанные спектры отражения воспроизводят основные спектральные особенности, наблюдаемые в экспериментальных спектрах нанокластеров $\mathrm{Au}$, их изменение с размером нанокластеров и толщиной слоя $\mathrm{SiO}_{2}$. Численная модель, используемая для подгонки экспериментальных спектров отражения, применялась для моделирования спектров поглощения и определения зависимости положения ЛППР как функции от размера и толщины слоя $\mathrm{SiO}_{2}$. Предлагаемый метод демонстрирует возможность применения спектроскопии отражения для анализа металлических нанокластерных массивов на непрозрачных подложках и потенциально может быть использован для экспрессного определения энергии ЛППР. Показано, что использование подложек $\mathrm{Si} / \mathrm{SiO}_{2}$ позволяет настраивать положение ЛППР в более широком спектральном диапазоне по сравнению со стандартными подложками из оксида кремния.

\section{Список литературы / References}

1. Monticone F., Alu A. Metamaterial, plasmonic and nanophotonic devices. In: Reports Prog. Phys., 2017, vol. 80. DOI 10.1088/1361-6633/aa518f

2. Guler U., Kildishev A. V., Boltasseva A., Shalaev V. M. Plasmonics on the slope of enlightenment: The role of transition metal nitrides. Faraday Discuss., 2015, vol. 178, p. 71-86. DOI $10.1039 / \mathrm{c} 4 \mathrm{fd} 00208 \mathrm{c}$

3. Mayer K. M., Hafner J. H., Antigen A. À. Localized surface plasmon resonance sensors. Chem. Rev., 2011, vol. 111, p. 3828-3857. DOI 10.1021/cr100313v

4. Kabashin A. V., Evans P., Pastkovsky S. et al. Plasmonic nanorod metamaterials for biosensing. Nat. Mater., 2009, vol. 8, p. 867-871. DOI 10.1038/nmat2546

5. Anker J. N., Hall W. P., Lyandres O. et al. Biosensing with plasmonic nanosensors. Nat. Mater., 2008, vol. 7, p. 442-453. DOI 10.1038/nmat2162

6. Stockman M. I. Nanoplasmonks: The physics behind the applications. Phys. Today, 2011, vol. 64, p. 39-44. DOI 10.1063/1.3554315

7. Gramotnev D. K., Bozhevolnyi S. I. Plasmonics beyond the diffraction limit. Nat. Photonics, 2010, vol. 4, p. 83-91. DOI 10.1038/nphoton.2009.282

8. Ozbay E. Plasmonics: Merging photonics and electronics at nanoscale dimensions. Science, 2006, vol. 311, p. 189-193. DOI 10.1126/science.1114849

9. Dionne J. A., Sweatlock L. A., Sheldon M. T. et al. Silicon-based plasmonics for on-chip photonics. IEEE J. Sel. Top. Quantum Electron., 2010, vol. 16, p. 295-306. DOI 10.1109/ JSTQE.2009.2034983 
10. Li E.-P., Chu H.-S. Plasmonic Nanoelectronics and Sensing. Cambridge Uni. Press, 2014.

11. Bohren C. F., Huffman D. R. Absorption and Scattering of Light by Small Particles. Wiley, New York, 1983.

12. Langhammer C., Kasemo B., Zorić I. Absorption and scattering of light by Pt, Pd, Ag, and Au nanodisks: Absolute cross sections and branching ratios. J. Chem. Phys., 2007, vol. 126, p. 1-11. DOI 10.1063/1.2734550

13. Zorić I., Kasemo B., Langhammer C., Zaaach M. Gold, Platinum, and Aluminum Nanodisk Plasmons: Material Damping Mechanisms. ACS Nano, 2011, vol. 5, p. 2535-2546. DOI 10.1021/ nn102166t

14. Qin F., Cui X., Ruan Q. et al. Role of shape in substrate-induced plasmonic shift and mode uncovering on gold nanocrystals. Nanoscale, 2016, vol. 8, p. 17645-17657. DOI 10.1039/ C6NR06387J

15. Leong K., Chen Y., Masiello D. J. et al. Cooperative near-field surface plasmon enhanced quantum dot nanoarrays. Adv. Funct. Mater., 2010, vol. 20, p. 2675-2682. DOI 10.1002/ adfm. 201000424

16. Sheremet E., Milekhin A. G., Rodriguez R. D. et al. Surface-and tip-enhanced resonant Raman scattering from CdSe nanocrystals. Phys. Chem., 2119, vol. 17, p. 21198-21203. DOI $10.1039 / \mathrm{c} 4 \mathrm{cp} 05087 \mathrm{~h}$

17. Gillibert R., Sarkar M., Bryche J. F. et al. Directional surface enhanced Raman scattering on gold nano-gratings. Nanotechnology, 2016, vol. 27. DOI 10.1088/0957-4484/27/11/115202

18. Milekhin A. G., Yeryukov N. A., Sveshnikova L. L. et al. Surface enhanced Raman scattering by organic and inorganic semiconductors formed on laterally ordered arrays of $\mathrm{Au}$ nanoclusters. Thin Solid Films, 2013, vol. 543, p. 35-40. DOI 10.1016/j.tsf.2013.03.070

19. Ovchinnikov $V$. Reflection from Irregular Array of Silver Nanoparticles on Multilayer Substrate. In: Proceedings of The Ninth International Conference on Quantum, Nano/Bio, and Micro Technologies (ICQNM 2015), 2015, p. 16-21. ISBN 978-1-61208-303-2

20. Johnson P. B., Christy R. W. Optical constants of the noble metals. Phys. Rev. B., 1972, vol. 6, p. 4370-4379. DOI 10.1103/PhysRevB.6.4370

21. Johnson P., Christy R. Optical constants of transition metals: Ti, V, Cr, Mn, Fe, Co, Ni, and Pd. Phys. Rev. B., 1974, vol. 9, p. 5056-5070. DOI 10.1103/PhysRevB.9.5056

22. Aspnes D. E., Studna A. A. Dielectric functions and optical parameters of Si, Ge, GaP, GaAs, GaSb, InP, InAs, and InSb from 1.5 to 6.0 eV. Phys. Rev. B., 1983, vol. 27, p. 985-1009. DOI 10.1103/PhysRevB.27.985

23. Malitson I. H. Interspecimen Comparison of the Refractive Index of Fused Silica. J. Opt. Soc. Am., 1965, vol. 55, p. 1205. DOI 10.1364/JOSA.55.001205

24. Hu E.-T., Liu X.-X., Cai Q.-Y. et al. Tunable optical properties of co-sputtered $\mathrm{Ti}^{-\mathrm{SiO}_{2}}$ nanocomposite thin films. Opt. Mater. Express, 2017, vol. 7, p. 2387. DOI 10.1364/OME. 7.002387

25. Kreibig U., Vollmer M. Optical Properties of Metal Clusters. Berlin, Springer, 1995.

26. Maier S. A., Kik P. G., Atwater H. A. et al. Local detection of electromagnetic energy transport below the diffraction limit in metal nanoparticle plasmon waveguides. Nat. Mater., 2003, vol. 2, p. 229-232. DOI 10.1038/nmat852

27. Maier S. A., Kik P. G., Atwater H. A. Observation of coupled plasmon-polariton modes in $\mathrm{Au}$ nanoparticle chain waveguides of different lengths: Estimation of waveguide loss. Appl. Phys. Lett., 2002, vol. 81, p. 1714-1716. DOI 10.1063/1.1503870 
28. Milekhin A. G., Yeryukov N. A., Sveshnikova L. L. et al. Combination of surface- and interference-enhanced Raman scattering by $\mathrm{CuS}$ nanocrystals on nanopatterned $\mathrm{Au}$ structures. Beilstein J. Nanotechnol., 2015, vol. 6, p. 749-754. DOI 10.3762/bjnano.6.77

29. Nicolas R., Lévêque G., Marae-Djouda J. et al. Plasmonic mode interferences and Fano resonances in Metal-Insulator-Metal nanostructured interface. Sci. Rep., 2015, vol. 5, p. 1-11. DOI 10.1038/srep14419

30. Ruemmele J. A., Hall W. P., Ruvuna L. K., Van Duyne R. P. A localized surface plasmon resonance imaging instrument for multiplexed biosensing. Anal. Chem., 2013, vol. 85, p. 45604566. DOI $10.1021 / \mathrm{ac} 400192 \mathrm{f}$

Материал поступил в редколлегию Received 08.02.2019

\section{Сведения об авторах / Information about the Authors}

Аникин Кирилл Витальевич, Институт физики полупроводников им. А. В. Ржанова СО РАН (пр. Академика Лаврентьева, 13, Новосибирск, 630090, Россия)

Kirill V. Anikin, A. V. Rzhanov Institute of Semiconductor Physics SB RAS (13 Academician Lavrentiev Ave., Novosibirsk, 630090, Russian Federation)

anikin@isp.nsc.ru

Милёхин Александр Германович, доктор физико-математических наук, Институт физики полупроводников им. А. В. Ржанова СО РАН (пр. Академика Лаврентьева, 13, Новосибирск, 630090, Россия); Новосибирский государственный университет (ул. Пирогова, 2, Новосибирск, 630090, Россия)

Aleksandr G. Milekhin, Doctor of Science (Physics and Mathematics), A. V. Rzhanov Institute of Semiconductor Physics SB RAS (13 Academician Lavrentiev Ave., Novosibirsk, 630090, Russian Federation); Novosibirsk State University (2 Pirogov Str., Novosibirsk, 630090, Russian Federation)

milekhin@isp.nsc.ru

Родякина Екатерина Евгеньевна, кандидат физико-математических наук, Институт физики полупроводников им. А. В. Ржанова СО РАН (пр. Академика Лаврентьева, 13, Новосибирск, 630090, Россия); Новосибирский государственный университет (ул. Пирогова, 2, Новосибирск, 630090, Россия)

Ekaterina E. Rodyakina, Candidate of Science (Physics and Mathematics), A. V. Rzhanov Institute of Semiconductor Physics SB RAS (13 Academician Lavrentiev Ave., Novosibirsk, 630090, Russian Federation); Novosibirsk State University (2 Pirogov Str., Novosibirsk, 630090, Russian Federation)

rodyakina@isp.nsc.ru

ORCID 0000-0003-4940-7074 
Вебер Сергей Леонидович, кандидат физико-математических наук, Институт «Международный томографический центр» СО РАН (ул. Институтская, За, Новосибирск, 630090, Россия)

Sergey L. Veber, Candidate of Science (Physics and Mathematics), International Tomography Center SB RAS (3a Institutskaya Str., Novosibirsk, 630090, Russian Federation)

Латышев Александр Васильевич, доктор физико-математических наук, академик РАН, профессор, Институт физики полупроводников им. А. В. Ржанова СО РАН (пр. Академика Лаврентьева, 13, Новосибирск, 630090, Россия); Новосибирский государственный университет (ул. Пирогова, 2, Новосибирск, 630090, Россия)

Aleksandr V. Latyshev, Doctor of Science (Physics and Mathematics), Academician of RAS, Professor, A. V. Rzhanov Institute of Semiconductor Physics SB RAS (13 Academician Lavrentiev Ave., Novosibirsk, 630090, Russian Federation); Novosibirsk State University (2 Pirogov Str., Novosibirsk, 630090, Russian Federation)

latyshev@isp.nsc.ru

Dietrich. R. T. Zahn, Professor, Technische Universität Chemnitz, Physics Department / Semiconductor Physics (70Reichenhainer Straße, 09126 Chemnitz)

zahn@physik.tu-chemnitz.de 